\title{
ERRATUM
}

\section{Erratum to: The Intensive Care Global Study on Severe Acute Respiratory Infection (IC-GLOSSARI): a multicenter, multinational, 14-day inception cohort study}

\author{
Yasser Sakr ${ }^{1 *}$, Ricard Ferrer ${ }^{2}$, Konrad Reinhart ${ }^{1}$, Richard Beale $^{3}$, Andrew Rhodes $^{4}$, Rui Moreno ${ }^{5}$, \\ Jean Francois Timsit ${ }^{6}$, Laurent Brochard ${ }^{7,8}$, B. Taylor Thompson ${ }^{9,10}$, Ederlon Rezende ${ }^{11}$, Jean Daniel Chiche ${ }^{12,13,14}$, \\ IC-GLOSSARI Investigators and ESICM Trials Group
}

(C) 2016 Springer-Verlag Berlin Heidelberg and ESICM

\section{Erratum to: Intensive Care Med DOI 10.1007/s00134-015-4206-2}

In the original publication the IC-GLOSSARI Investigators were presented in a way that did not permit their listing as collaborators in Medline. This error by the publisher has now been corrected.

\section{Author details}

${ }^{1}$ Department of Anesthesiology and Intensive Care, Uniklinikum Jena, Erlanger Allee 103, 07743 Jena, Germany. ${ }^{2}$ Intensive Care Department, Hospital Universitari Vall d'Hebron, CIBER Enfermedades Respiratorias, Passeig de la Vall d'Hebron, 119-129, 08035 Barcelona, Spain. ${ }^{3}$ King's Health Partners, Westminster Bridge Road, London SE1 7EH, UK. ${ }^{4}$ Critical Care Directorate, St George's University Hospitals NHS Foundation Trust, London, UK. ${ }^{5}$ Intensive Care Unit, Hospital de São Jos, Unidade de Cuidados Intensivos Neurocríticos,
Centro Hospitalar de Lisboa Central, EPE, Rua José António Serrano, S, 1150-199 Lisbon, Portugal. ${ }^{6}$ Medical ICU, Paris Diderot University/Bichat Teaching Hospital Medical and Infectious Diseases ICU, 46 rue Henri Huchard, Paris 75018, France. ${ }^{7}$ Interdepartmental Division of Critical Care Medicine, University of Toronto, Toronto, Canada. ${ }^{8}$ Keenan Research Centre, Li Ka Shing Knowledge Institute, St. Michael's Hospital, 30 Bond Street, Toronto, ON M5B 1W8, Canada. ${ }^{9}$ Division of Pulmonary and Critical Care Medicine, Massachusetts General Hospital, Boston, USA. ${ }^{10}$ Harvard Medical School, 55 Fruit St, Boston, MA 02114, USA. ${ }^{11}$ Serviço de Terapia intensiva na IAMSPE-Instituto de Assistência Médica ao Servidor Público Estadual, Av. Ibirapuera, 981, São Paulo, SP 04028-000, Brazil. ${ }^{12}$ Medical Intensive Care Unit, Paris Descartes University, Paris, France. ${ }^{13}$ Hôpital Cochin, Paris, France. ${ }^{14}$ Department of Cell Biology and Host-Pathogen Interaction, Cochin Institute, 27 Rue du Faubourg St Jacques, 75679 Paris Cedex 14, France.

Published online: 1 April 2016

*Correspondence: yasser.sakr@med.uni-jena.de

1 Department of Anesthesiology and Intensive Care, Uniklinikum Jena, Erlanger Allee 103, 07743 Jena, Germany

Full author information is available at the end of the article

The online version of the original article can be found under doi:10.1007/ s00134-015-4206-2.

\section{焦

